\title{
Noise-Induced Truth Seeking of Heterogeneous Hegselmann-Krause Opinion Dynamics
}

\author{
Yan Liu ${ }^{10}{ }^{1,2}$ and Lipo Mo $\mathbb{i D}^{3}$ \\ ${ }^{1}$ School of Automation and Electrical Engineering, University of Science and Technology, Beijing 100083, China \\ ${ }^{2}$ Key Laboratory of Knowledge Automation for Industrial Processes, Ministry of Education, Beijing 100083, China \\ ${ }^{3}$ School of Science, Beijing Technology and Business University, Beijing 100048, China \\ Correspondence should be addressed to Lipo Mo; beihangmlp@126.com
}

Received 6 September 2018; Revised 10 October 2018; Accepted 25 October 2018; Published 11 November 2018

Academic Editor: Carlo Bianca

Copyright (C) 2018 Yan Liu and Lipo Mo. This is an open access article distributed under the Creative Commons Attribution License, which permits unrestricted use, distribution, and reproduction in any medium, provided the original work is properly cited.

\begin{abstract}
In this paper, we study the noise-induced truth seeking for heterogeneous Hegselmann-Krause (HK) model in opinion dynamics. It has been proved that small noise could induce the group to achieve truth in homogeneous HK model; however, for the more practical heterogeneous HK model, the theoretical conclusion is absent. Here, we prove that small noise could also induce the group to achieve truth in heterogenous HK model, and, moreover, we first theoretically prove that large noise could drive some agents to deviate from the truth. These theoretical findings evidently reveal how the free information flow spreading in the media determines the social truth seeking.
\end{abstract}

\section{Introduction}

Recently opinion dynamics has attracted increasing attention from researchers of various fields [1-6]. In opinion dynamics, a basic model is the widely known Hegselmann-Krause (HK) model, which is built based on the so-called bounded confidence mechanism [7-9]. In HK model, each agent possesses a confidence threshold and updates its opinion by averaging those of its neighbors (agents whose opinions locate within its confidence threshold). If all the agents have an identical confidence threshold, the model is said to be homogeneous; otherwise, the model is said to be heterogeneous. Though it looks simple, the HK dynamics captures some basic opinion phenomena such as consensus and cleavage and also provides a fundamental mechanism that could develop more opinion models [10-12].

In social dynamics, how the social groups seek and achieve truth interests much studies. In [13], a truth seeking model was built based on the HK dynamics. Using the model, some factors are found to be related to whether the group achieves the truth. One is the number of truth seekers in the group. If all the agents in the group are truth seekers, it is proved that the group will finally achieve the truth. If there are agents which are not truth seekers in the group, whether the truth can be acquired is highly dependent on the initial conditions of the group and most likely the truth cannot be achieved by the whole group. Very lately, using the model in [13] a study found that noise plays a dominant role for the group to achieve the truth [14]. It is rigorously proved that, in the presence of small noise, the group will almost surely achieve the truth for any initial conditions and even there is only one truth seeker in the group. The noise is appropriately explained as the intensive free information we randomly received from media each day in this information era, and the finding actually reveals that free information flow spreading in the media benefits the truth seeking in a society.

The truth seeking model in [14] is homogeneous; i.e., all the agents possess an identical confidence threshold and all the truth seekers have the same preference for the truth. However, as we know, the real agents in a society are usually heterogeneous and have different confidence threshold and different preference for truth. Moreover, [14] only reveals the influence of properly small noise which could induce the truth seeking. But, in real social network, there always exist some "strong" noises, such as some fabricated rumors which may violently perturb people's opinion. Thus, there is a great 
need to investigate theoretically how small and large noise determine the truth seeking of heterogeneous HK model.

In this paper, we completely establish the theoretical analysis for the truth seeking of heterogeneous HK model with noise. Similar to [14], we also prove that small noise could drive the group to the truth from any initial state; what is more important, we for the first time rigorously prove that when noise is large, the truth cannot be achieved in the group no matter what the initial state is. These results reveal completely how free information flow determines the truth seeking in social network.

The rest of this paper is organized as follows: Section 2 gives the heterogeneous truth seeking model with noise; Section 3 proves the main theoretical results about how small and large noise determine the truth seeking; Section 4 provides some simulation studies to verify the main theoretical results; and at last Section 5 concludes the paper.

\section{Preliminary and Formulation}

2.1. Heterogeneous Truth Seeking Model. Let $\mathcal{S}=\{1, \ldots, n\}$ be a group of agents which consist of truth seekers and neutral followers and $\mathscr{K} \subset \mathcal{S}$ be the set of truth seekers. Then the heterogeneous truth seeking model is modelled as

$$
\begin{aligned}
y_{i}(t+1)= & \lambda_{i} I_{\{i \in \mathscr{K}\}} Q \\
& +\left(1-\lambda_{i} I_{\{i \in \mathscr{K}\}}\right) \sum_{j \in \mathcal{N}_{i}(y(t))} \frac{y_{j}(t)}{\left|\mathcal{N}_{i}(y(t))\right|} \\
& +\eta_{i}(t+1), \quad i \in \mathcal{S},
\end{aligned}
$$

where $y_{i}(t) \in[0,1]$ is the state of agent $i$ at time $t, Q \in[0,1]$ is the truth value, $\lambda_{i} \in[0,1]$ is the attraction strength of truth $Q$ for agent $i$, and

$$
\mathcal{N}_{i}(y(t))=\left\{j \in \mathcal{S}|| y_{j}(t)-y_{i}(t) \mid \leq r_{i}\right\}
$$

is the neighbor set of $i$ at time $t$ with $r_{i} \in(0,1]$ being the confidence threshold. In addition, $I_{\{i \in \mathscr{K}\}}$ is the indicator function which takes value 1 or 0 according to $i \in \mathscr{K}$ or not and $|\cdot|$ represents the absolute value of a real number or the cardinal number of a set accordingly. $\left\{\xi_{i}(t)\right\}_{i \in \mathcal{S}, t>0}$ are noises which are independent and uniformly distributed on $[-\delta, \delta]$ with $\delta>0$. If $r_{i}=r \in(0,1], \lambda_{i}=\lambda \in[0,1]$ for all $i \in \mathcal{S}$, the heterogeneous model (1) degenerates to a homogeneous one studied in [14]. For a truth seeker $i$, it has $\lambda_{i}>0$; i.e., $\mathscr{K}=\left\{i \in \mathcal{S}: 0<\lambda_{i} \leq 1\right\}$, while a neutral follower has $\lambda_{i}=0$.

In system (1), noise usually models the free information flow spread in social network. Each day we are exposed to intensive free information which will affect our opinions. However, we do not know what information we will meet, whether and how much they will change our opinions. This results in a stochastic effect of the update of our opinions. Mathematically, the introduction of noise will make opinion values exceed the bounded opinion space $[0,1]$. Similar to previous studies, we also make an assumption that the noisy opinion values are limited in $[0,1]$. Subsequently, model (1) is modified as

$$
y_{i}(t+1)= \begin{cases}1, & y_{i}^{*}(t)>1 \\ y_{i}^{*}(t), & y_{i}^{*}(t) \in[0,1] \\ 0, & y_{i}^{*}(t)<0\end{cases}
$$

$$
\forall i \in \mathcal{S}, t \geq 0
$$

where

$$
\begin{aligned}
y_{i}^{*}(t)= & \lambda_{i} I_{\{i \in \mathscr{K}\}} Q \\
& +\left(1-\lambda_{i} I_{\{i \in \mathscr{K}\}}\right) \sum_{j \in \mathscr{N}_{i}(y(t))} \frac{y_{j}(t)}{\left|\mathcal{N}_{i}(y(t))\right|} \\
& +\eta_{i}(t+1) .
\end{aligned}
$$

and

$$
\begin{aligned}
& 1 \leq|\mathscr{K}| \leq n, \\
& \left\{\eta_{i}(t), i \in \mathcal{S}, t\right. \\
& \quad>0\} \text { are independent and uniformly distributed on }[-\delta, \delta] .
\end{aligned}
$$

2.2. Achieving Truth. The definition of achieving truth for the noisy system (3)-(5) is as follows.

Definition 1 (see [14]). Let $d_{\mathcal{S}}^{\mathrm{Q}}(t)=\max _{i \in \mathcal{S}}\left|y_{i}(t)-\mathrm{Q}\right|$; if $\lim \sup _{t \rightarrow \infty} d_{\delta}^{\mathrm{Q}}(t) \leq \phi$ a.s., we say system (3)-(5) achieves the truth $Q$ with $\phi$-precision.

\section{Main Results}

3.1. Small Noise Benefits Truth Seeking. In [14], it was proved that small noise could drive the homogeneous HK model to achieve the truth. In this part, we will show how small noise affects the truth seeking of heterogeneous HK model. Actually, we obtain the same conclusion for the heterogeneous $\mathrm{HK}$ model.

Let $m=|\mathscr{K}|, \underline{\lambda}=\min _{i \in \mathcal{S}} \lambda_{i}, \underline{r}=\min _{i \in \mathcal{S}} r_{i}$ and denote

$$
\begin{aligned}
\delta_{1} & =\frac{n(1-\underline{\lambda})}{m \underline{\lambda}} \delta+\delta, \\
\delta_{2} & =\frac{n}{m \underline{\lambda}} \delta+\delta \\
\bar{\delta} & =\max \left\{\delta_{1}, \delta_{2}\right\}, \\
\underline{\delta} & =\min \left\{\frac{m \underline{\lambda}}{2 n+(2 m-n) \underline{\lambda}} \underline{r}, \frac{m}{n+2 m} \underline{r}\right\},
\end{aligned}
$$

and then, for system (3)-(5), we have the following main result.

Theorem 2. Let $0<\underline{\lambda}<1$ in model (3)-(5), and then, for any initial state $y_{i}(0) \in[0,1], r_{i} \in(0,1], i \in \mathcal{S}$ and $\delta \in(0, \underline{\delta}]$, the model will a.s. achieve the truth $Q$ with $\bar{\delta}$-precision. 
Also, to demonstrate Theorem 2 better, we present the following corollary like that in [14].

Corollary 3. Suppose $m=\lceil n / 2\rceil, \underline{\lambda}=0.5$, and then a.s. $\lim \sup _{t \rightarrow \infty} d_{\mathcal{S}}^{\mathrm{Q}}(t) \leq 5 \delta$ for all $\delta \in(0, \underline{r} / 8]$.

The key of the proof of Theorem 2 is the following lemma.

Lemma 4. Let $0<\underline{\lambda} \leq 1$, and if there is a moment $t_{0}<\infty$ a.s. such that $d_{\mathscr{K}}^{\mathrm{Q}}\left(t_{0}\right) \leq \bar{\delta}_{1}, d \frac{\mathrm{Q}}{\mathscr{K}}\left(t_{0}\right) \leq \delta_{2}(\overline{\mathscr{K}}=\mathcal{S}-\mathscr{K})$, then, for $0<$ $\delta \leq \underline{\delta}$, it holds $\lim \sup _{t \rightarrow \infty} d_{\mathscr{K}}^{\mathrm{Q}}(t) \leq \delta_{1}, \lim \sup _{t \rightarrow \infty} d \frac{d_{\mathscr{K}}^{\mathrm{Q}}}{(t) \leq}$ $\delta_{2}$ a.s.

Proof. It can be checked that, at moment $t_{0}$, it holds that

$$
\begin{aligned}
\max _{i, j \in \mathcal{S}}\left|y_{i}\left(t_{0}\right)-y_{j}\left(t_{0}\right)\right| \leq & \max _{i \in \mathcal{S}}\left|y_{i}\left(t_{0}\right)-Q\right| \\
& +\max _{j \in \mathcal{S}}\left|y_{j}\left(t_{0}\right)-Q\right| \leq \delta_{1}+\delta_{2} \\
& =\frac{2 n+(2 m-n) \underline{\lambda}}{m \underline{\lambda}} \leq \underline{r} .
\end{aligned}
$$

Thus all agents are neighbors to each other at moment $t_{0}$ and hence $\left|\mathcal{N}_{i}\left(y\left(t_{0}\right)\right)\right|=n, i \in \mathcal{S}$. By (3) and (4), it has

$$
\begin{aligned}
y_{i}\left(t_{0}+1\right)= & \lambda_{i} Q+\left(1-\lambda_{i}\right) \frac{\sum_{k \in \mathcal{S}} y_{k}\left(t_{0}\right)}{n} \\
& +\eta_{i}\left(t_{0}+1\right), \quad i \in \mathscr{K}, \\
y_{j}\left(t_{0}+1\right)= & \frac{\sum_{k \in \mathcal{S}} y_{k}\left(t_{0}\right)}{n}+\eta_{j}\left(t_{0}+1\right), \quad j \in \overline{\mathscr{K}} .
\end{aligned}
$$

Thus

$$
\begin{aligned}
& \left|y_{i}\left(t_{0}+1\right)-Q\right| \\
& \quad=\left|\lambda_{i} Q-Q+(1-\lambda) \frac{\sum_{k \in \mathcal{S}} y_{k}\left(t_{0}\right)}{n}+\eta_{i}\left(t_{0}+1\right)\right| \\
& \quad \leq \frac{1-\lambda_{i}}{n} \sum_{k \in \mathcal{S}}\left|Q-y_{k}\left(t_{0}\right)\right|+\delta \\
& \quad \leq \frac{1-\underline{\lambda}}{n}\left(m \delta_{1}+(n-m) \delta_{2}\right)+\delta=\frac{1-\underline{\lambda}}{n} \frac{n^{2} \delta}{m \underline{\lambda}}+\delta \\
& \quad=\delta_{1}, \quad i \in \mathscr{K} .
\end{aligned}
$$

And also

$$
\begin{aligned}
\left|y_{j}\left(t_{0}+1\right)-Q\right| & =\left|\frac{\sum_{k \in \mathcal{S}} y_{k}\left(t_{0}\right)}{n}-Q+\eta_{i}\left(t_{0}+1\right)\right| \\
& \leq \frac{1}{n} \sum_{k \in \mathcal{S}}\left|Q-y_{k}\left(t_{0}\right)\right|+\delta \\
& \leq \frac{m \delta_{1}+(n-m) \delta_{2}}{n}+\delta=\frac{n \delta}{m \underline{\lambda}}+\delta \\
& =\delta_{2}, \quad j \in \overline{\mathscr{K}} .
\end{aligned}
$$

Consequently

$$
\begin{aligned}
& d_{\mathscr{K}}^{\mathrm{Q}}\left(t_{0}+1\right) \leq \delta_{1}, \\
& d \frac{\mathrm{Q}}{\mathscr{K}}\left(t_{0}+1\right) \leq \delta_{2} .
\end{aligned}
$$

Repeating the above procedure obtains the conclusion.

The proof of Lemma 4 quite follows the line of Lemma 3.2 in [14]. It tells that there is an "attraction region" for the system to achieve the truth, and once the opinion of the group enters the region, small noise cannot drive them out. The main point of the proof of Lemma 4 is to find the "attraction region" in the heterogeneous model. With Lemma 4, the rest of the proof of Theorem 2 is similar to that of Theorem 3.1 in [14] and we omit it here.

3.2. Large Noise Harms Truth Seeking. Theorem 2 reveals that small noise could promote the truth seeking of the heterogeneous $\mathrm{HK}$ model. The noise in social networks mainly models the free information flow spreading in social media that influences people's opinion every day. Usually most information softly changes our opinions and brings small noise to the opinion dynamics; however, there are always some breaking news or rumors in social networks that could change our opinion shockingly and bring severe noise to the opinion dynamics. It is absent in [14] how large noise or breaking information affects the truth seeking in opinion dynamics. Here, we for the first time investigate this problem with the heterogeneous HK model and reveal theoretically that large noise is detrimental to the truth seeking of opinion dynamics.

Theorem 5. Suppose $|\mathscr{K}|<n$ in system (3)-(5) and $\underline{r}^{\prime}<\bar{D}$, where $\underline{r}^{\prime}=\min _{i \in \mathscr{K}} r_{i}, \bar{D}=\max \{Q, 1-Q\}$ with $Q \in[0,1]$. If $\delta>\underline{r}^{\prime}$, then, for all $y(0) \in[0,1]^{n}$, it has $d_{\delta}^{\mathrm{Q}}=\bar{D}$.

Remark 6. In Theorem 5, we suppose $|\mathscr{K}|<n$; i.e., there exist agents which are not truth seekers in the system. If all agents are truth seekers, it can be proved that the opinions will stay near the truth value $Q$ within an area dependent on the noise strength $\delta$. However, if there are neutral followers, Theorem 5 actually implies that some opinions will deviate from the truth value with any far distance within the opinion space. Theorem 2 shows an order phenomenon when noise is small while Theorem 5 shows a disorder phenomenon when noise is large. The measure of order is by the $\phi$ precision in Definition 1 and Theorem 2 reveals that when noise strength diminishes the precision increases. Theorem 5 shows that when large noise makes a disorder, the precision is independent of the noise strength and can be worst.

Proof. Let $Q \in[0,1 / 2]$ without loss of generality, and then we only need to prove that there exists $i \in \mathcal{S}$ such that $\lim \sup _{t \rightarrow \infty} y_{i}(t)=1$ a.s.; i.e.

$$
P\left\{\bigcup_{t_{0}=0}^{\infty}\left\{y_{i}(t)<1, t>t_{0}\right\}\right\}=0 .
$$


Given any $t_{0} \geq 0$ consider the following noise protocol: for $t \geq t_{0}$,

$$
\eta_{j}(t) \in\left(\frac{\delta+\underline{r}^{\prime}}{2}, \delta\right], \quad j \in \mathcal{S}
$$

Let $\mathscr{M}=\left\{i \in \overline{\mathscr{K}} \mid r_{i}=\min _{j \in \overline{\mathscr{K}}} r_{j}\right\}$, and then, for $i \in \mathscr{M}$, by (4), it has under protocol (13) that

$$
\begin{aligned}
y_{i}^{*}\left(t_{0}+1\right) & =\frac{\sum_{k \in \mathcal{N}_{i}\left(y\left(t_{0}\right)\right)} y_{k}\left(t_{0}\right)}{\left|\mathcal{N}_{i}\left(y\left(t_{0}\right)\right)\right|}+\eta_{i}\left(t_{0}+1\right) \\
& >\frac{\sum_{k \in \mathcal{N}_{i}\left(y\left(t_{0}\right)\right)} y_{k}\left(t_{0}\right)}{\left|\mathcal{N}_{i}\left(y\left(t_{0}\right)\right)\right|}+\frac{\delta+\underline{r}^{\prime}}{2}, \quad t \geq 0 .
\end{aligned}
$$

If $y_{i}^{*}\left(t_{0}\right) \geq 1$, it has $y_{i}\left(t_{0}+1\right)=1$; otherwise, $y_{i}\left(t_{0}+1\right)=$ $y_{i}^{*}\left(t_{0}+1\right)<1$. Since $\left|y_{k}\left(t_{0}\right)-y_{i}\left(t_{0}\right)\right| \leq \underline{r}^{\prime}$, for $k \in \mathcal{N}_{i}\left(y\left(t_{0}\right)\right)$, by (14)

$$
\begin{aligned}
y_{i}\left(t_{0}+1\right)-y_{i}\left(t_{0}\right)> & \frac{\sum_{k \in \mathcal{N}_{i}\left(y\left(t_{0}\right)\right)}\left(y_{k}\left(t_{0}\right)-y_{i}\left(t_{0}\right)\right)}{\left|\mathcal{N}_{i}\left(y\left(t_{0}\right)\right)\right|} \\
& +\frac{\delta+\underline{r}^{\prime}}{2} \geq-\underline{r}^{\prime}+\frac{\delta+\underline{r}^{\prime}}{2} \\
= & \frac{\delta-\underline{r}^{\prime}}{2}
\end{aligned}
$$

This implies, at moment $t_{0}+1$, the opinion value of agent $i$ will increase by no less than $\left(\delta-\underline{r}^{\prime}\right) / 2$ under protocol (13). Note that $P\left\{\eta_{j}(t) \in\left(\left(\delta+\underline{r}^{\prime}\right) / 2, \delta\right]\right\}=\left(\delta-\underline{r}^{\prime}\right) / 4 \delta$ for all $j \in \mathcal{S}, t \geq t_{0}$, and, by independence of $\eta_{i}(t)$, it has

$$
P\left\{y_{i}\left(t_{0}+1\right)-y_{i}\left(t_{0}\right)>\frac{\delta-\underline{r}^{\prime}}{2}\right\} \geq\left(\frac{\delta-\underline{r}^{\prime}}{4 \delta}\right)^{n}>0
$$

Denote $L=\left\lceil 2 /\left(\delta-\underline{r}^{\prime}\right)\right\rceil$ and repeat the above procedure for $L$ times, and we know that, under protocol (13), $y_{i}\left(t_{0}+L\right)=1$. By (16) and with independence of $\eta_{i}(t), i \in \mathcal{S}, t \geq 1$, it has

$$
P\left\{y_{i}\left(t_{0}+L\right)=1\right\} \geq\left(\frac{\delta-\underline{r}^{\prime}}{4 \delta}\right)^{n L}>0
$$

and thus

$$
P\left\{y_{i}\left(t_{0}+L\right)<1\right\}<1-\left(\frac{\delta-\underline{r}^{\prime}}{4 \delta}\right)^{n L}<1 .
$$

Similarly, for all $t>t_{0}$

$$
P\left\{y_{i}(t)<1 \mid \bigcap_{t_{0} \leq l<t}\left\{y_{i}(l)<1\right\}\right\} \leq 1-\left(\frac{\delta-\underline{r}^{\prime}}{4 \delta}\right)^{n L} .
$$

Note that $P\left\{y_{i}\left(t_{0}\right)<1\right\} \leq 1$, and it has

$$
\begin{aligned}
& P\left\{y_{i}(t)<1, t>t_{0}\right\}=P\left\{\bigcap_{t=t_{0}+1}^{\infty}\left\{y_{i}(t)<1\right\}\right\} \\
& =\lim _{m \rightarrow \infty} P\left\{\bigcap_{t=t_{0}+1}^{m}\left\{y_{i}(t)<1\right\}\right\} \\
& \leq \lim _{m \longrightarrow \infty} \prod_{t=t_{0}+1}^{m} P\left\{y_{i}(t)<1 \mid \bigcap_{t_{0} \leq l<t}\left\{y_{i}(l)<1\right\}\right\} \\
& \quad \cdot P\left\{y_{i}\left(t_{0}\right)<1\right\} \leq \lim _{m \longrightarrow \infty}\left(1-\left(\frac{\delta-\underline{r}}{4 \delta}\right)^{n L}\right)^{m} \\
& =0 .
\end{aligned}
$$

This completes the proof.

\section{Simulations}

In this section, we provide some simulation results to demonstrate the effect of small and large noise on truth seeking. Theorem 2 reveals that the phenomena of noise-induced truth seeking are independent of the group size $n$, the number of truth seekers $0<m \leq n$, and the truth value $Q$ and can be observed as long as the noise strength is properly small. Take $n=120$ agents, and 30 agents are truth seekers. The truth value $Q=0.5$, and the initial opinion values $y(0)=\left[y_{1}(0), \ldots, y_{120}(0)\right]$ are randomly generated on $[0,1]$, the heterogeneous confidence thresholds $r=\left[r_{1}, \ldots, r_{120}\right]$ are randomly generated on $(0,0.2]$, and the truth attraction strength $\lambda=\left[\lambda_{1}, \ldots, \lambda_{30}\right]$ is randomly generated on $[0,1]$. Figure 1 shows when there is no noise, only part of agents achieve the truth while some agents stay away from the truth. Then we add small noise with strength $\delta=0.0008$ to the group, and Figure 2 shows that all the agents finally achieve the truth. Next we add large noise $\delta=0.02$ to the group, and Figure 3 clearly shows that some agents frequently deviate from the truth value.

\section{Conclusions and Discussions}

In this paper, we investigated the noise-induced truth seeking of the heterogeneous HK model. Compared to the existing results about the homogeneous case, we completely revealed how the free information flow with different strength affects the truth seeking in the heterogeneous model. On the one hand we showed that noise with proper strength will almost surely bring the group to achieve the truth, while, on the other hand, we further proved that large noise, which implies drastic information perturbation, will make some agents deviate from the truth. These results not only reveal the role free information flow plays in the social truth seeking but also provide prospective intervention design for promoting the truth seeking of social group.

At last we want to have a further discussion for the order/disorder transition which appears in this paper. It can 


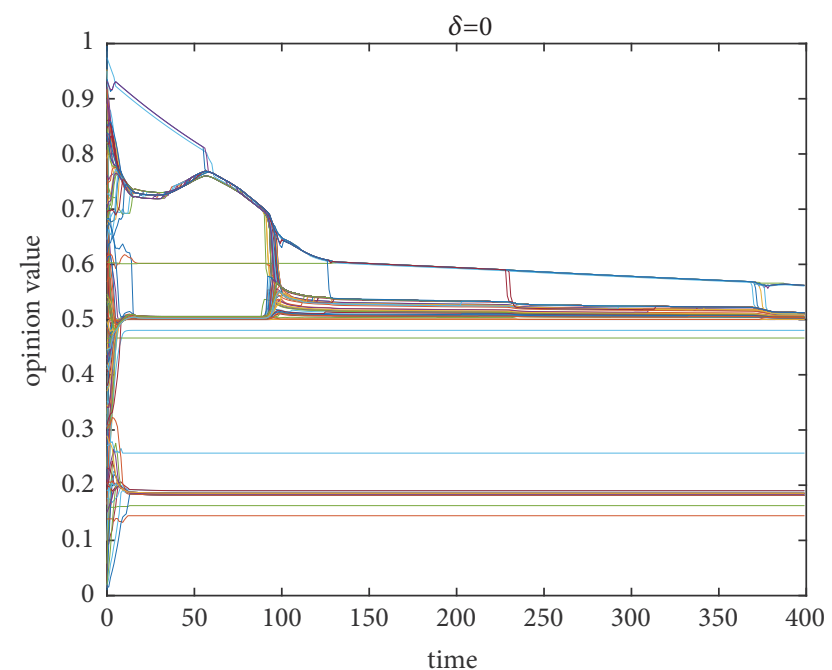

FIGURE 1: The truth seeking of model (3)-(5) without noise. It shows that some agents acquire the truth while the others stay away from the truth.

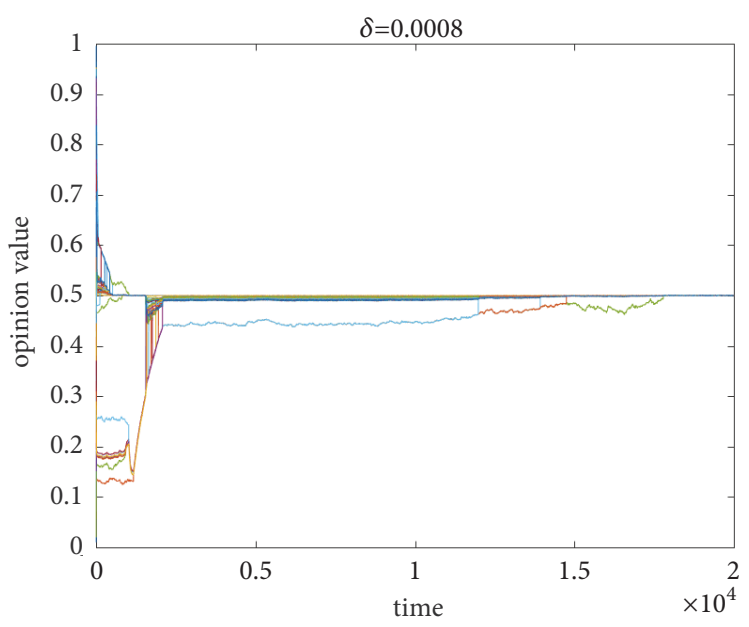

Figure 2: The truth seeking of model (3)-(5) with small noise. Here the noise strength is taken $\delta=0.0076$ and it shows that all the agents finally achieve the truth.

be clearly found that an order/disorder transition happens with the variation of noise strength. However, whether the transition is a critical or just a crossover phenomenon is yet unclear in this study. In [11], a critical noise strength is obtained by analysis for the homogeneous HK model, but the analysis for the heterogeneous case is far more complicated. Simulation studies can show that the noise strength bound for order ( $\delta$ in Theorem 2 ) and for disorder ( $\delta$ in Theorem 5 ) is conservative and a fuzzy region of noise strength exists between the noise strength bounds of order and disorder.

\section{Data Availability}

No data were used to support this study.

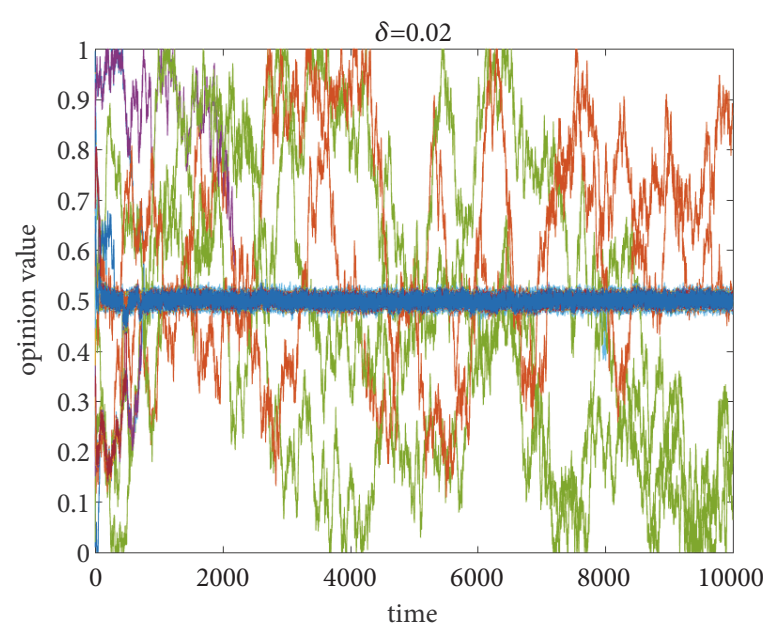

FIgURE 3: The truth seeking of model (3)-(5) with large noise. The noise strength is taken $\delta=0.045$ and it shows that the truth cannot be achieved in the group while some agents frequently deviate from the truth.

\section{Conflicts of Interest}

The authors declare that there are no conflicts of interest regarding the publication of this paper.

\section{Acknowledgments}

This work is supported by the General Plan of Scientific Research Project of Beijing Educational Committee and the National Natural Science Foundation (NNSF) of China (Grant no. 61304155).

\section{References}

[1] C. Castellano, S. Fortunato, and V. Loreto, "Statistical physics of social dynamics," Reviews of Modern Physics, vol. 81, no. 2, pp. 591-646, 2009.

[2] S. Galam, Sociophysics-A Physicist's Modeling of Psycho-political Phenomena, Springer, New York, NY, USA, 2012.

[3] P. Sen and B. K. Chakrabarti, Sociophysics: An Introduction, Oxford University Press, 2013.

[4] A. V. Proskurnikov and R. Tempo, "A tutorial on modeling and analysis of dynamic social networks. Part I," Annual Reviews in Control, vol. 43, pp. 65-79, 2017.

[5] A. V. Proskurnikov and R. Tempo, "A tutorial on modeling and analysis of dynamic social networks. Part II," Annual Reviews in Control, vol. 45, pp. 166-190, 2018.

[6] Yong Ding and Lipo Mo, "Improved finite time in eliminating disagreement of opinion dynamics via noise," Advances in Mathematical Physics, vol. 2017, Article ID 1098169, 6 pages, 2017.

[7] G. Deffuant, D. Neau, F. Amblard, and G. Weisbuch, "Mixing beliefs among interacting agents," Advances in Complex Systems (ACS), vol. 3, no. 1, pp. 87-98, 2000.

[8] U. Krause, "A discrete nonlinear and non-autonomous model of consensus formation," in Communications in Difference Equations, S. Elaydi, G. Ldas, J. Popenda, and J. Rakowski, Eds., pp. 227-238, Gordon and Breach, Amsterdam, 2000. 
[9] R. Hegselmann and U. Krause, "Opinion dynamics and bounded confidence models, analysis, and simulation," Artificial Societies and Social Simulation, vol. 5, no. 3, pp. 1-33, 2002.

[10] V. D. Blondel, J. M. Hendrickx, and J. N. Tsitsiklis, "On Krause's multi-agent consensus model with state-dependent connectivity," IEEE Transactions on Automatic Control, vol. 54, no. 11, pp. 2586-2597, 2009.

[11] W. Su, G. Chen, and Y. Hong, "Noise leads to quasi-consensus of Hegselmann-Krause opinion dynamics," Automatica, vol. 85, pp. 448-454, 2017.

[12] W. Su, J. Guo, X. Chen, and G. Chen, Robust Fragmentation Modeling of Hegselmann-Krause-Type Dynamics, 2018.

[13] R. Hegselmann and U. Krause, "Truth and cognitive division of labour: first steps towards a computer aided social epistemology," Journal of Artificial Societies and Social Simulation, vol. 9, no. 3, pp. 1-28, 2006.

[14] W. Su and Y. Yu, "Free information flow benefits truth seeking," Journal of Systems Science \& Complexity, vol. 31, no. 4, pp. 964974, 2018. 


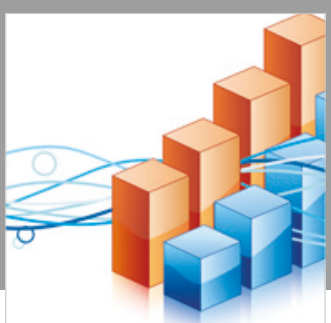

Advances in

Operations Research

\section{-n-m}
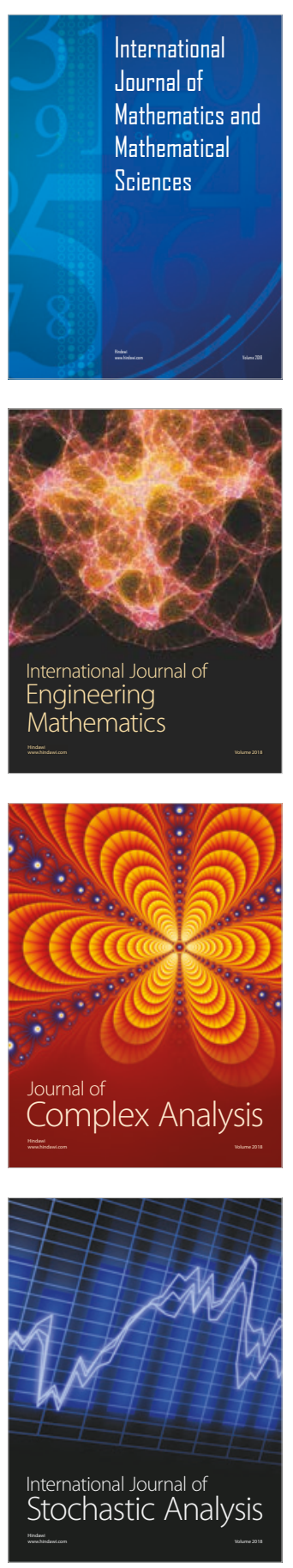
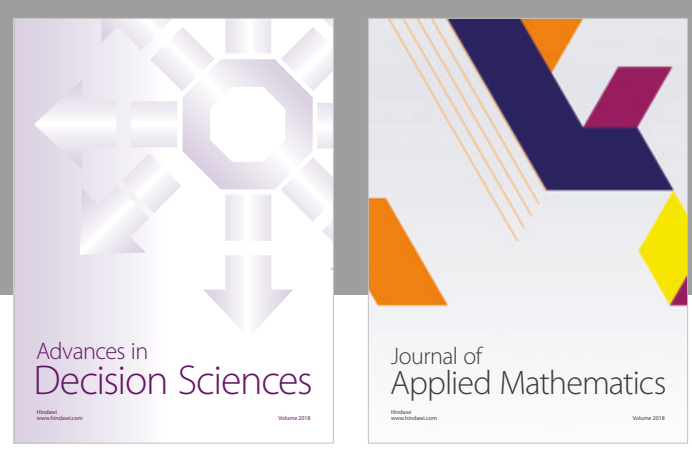

Journal of

Applied Mathematics
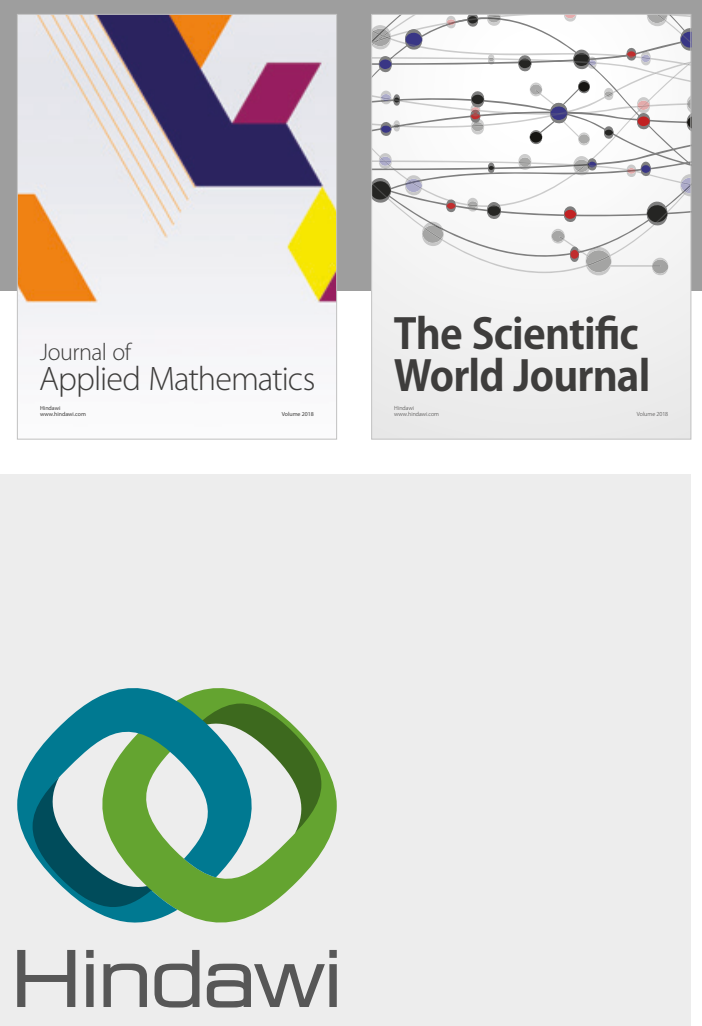

Submit your manuscripts at

www.hindawi.com

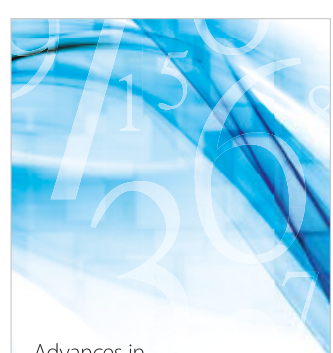

Advances in
Numerical Analysis
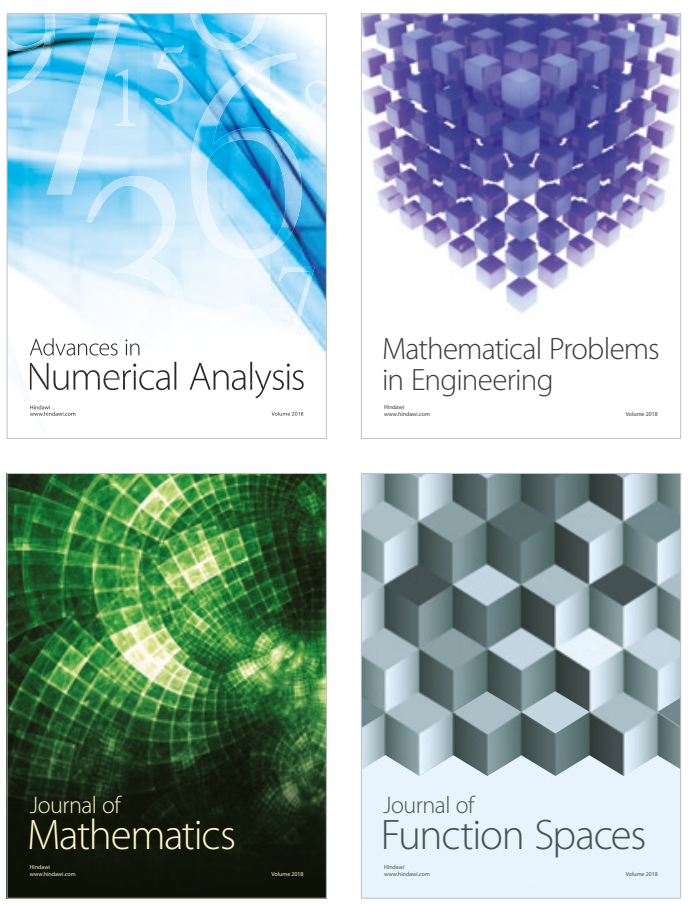

Mathematical Problems in Engineering

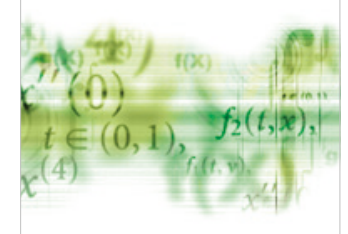

International Journal of

Differential Equations

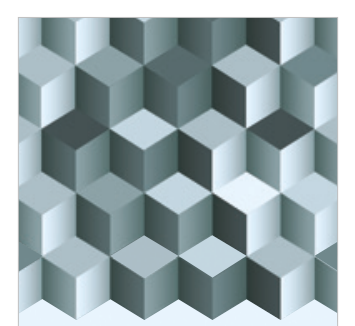

Journal of

Function Spaces

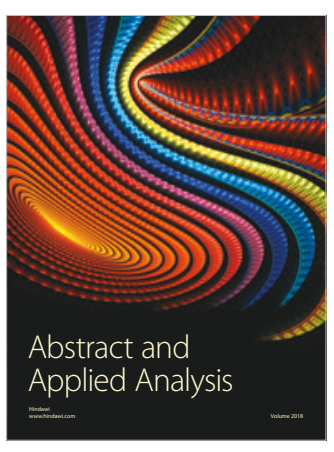

The Scientific

World Journal

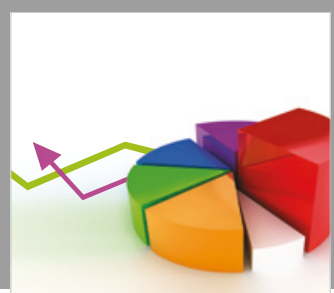

Journal of

Probability and Statistics
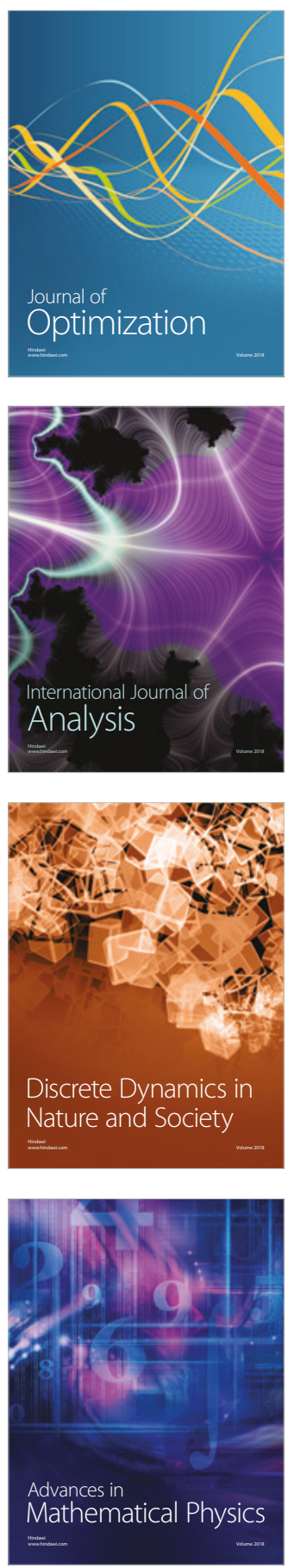